\title{
Perioperative risk factors of psychological distress in patients undergoing treatment for esophageal cancer
}

Yu Ohkura ${ }^{1,2,3^{*}}$ D, Junichi Shindoh ${ }^{2,3}$, Kanako Ichikura ${ }^{4}$, Harushi Udagawa ${ }^{2,3}$, Masaki Ueno $^{2,3}$ and Eisuke Matsushima ${ }^{1}$

\begin{abstract}
Background: Esophageal cancer patients often feel depressed and are fearful of metastasis and death. The objective of this study was to clarify the characteristics of patients with psychological distress at all 5 time points compared with patients with no psychological distress especially from standpoints of personal coping styles and QOL.
\end{abstract}

Methods: In total, 102 of 152 consecutive patients who attended the outpatient clinic at Toranomon Hospital between April 2017 and April 2019 met eligibility criteria for inclusion in this study. Questionnaires designed to identify psychological distress (HADS-scores) and assess QOL (EORTC QLQ C-30/OES18) were administered at 5 time points from the time of the first outpatient consultation to 3 months after esophagectomy. The questionnaire of coping strategies (MAC-scales) was administered at only time 1 point.

Results: Based on the trends of HADS-scores, we defined two groups: "persistent high-HAD scores" and "persistent low-HADS scores." There are strong relationships between psychological distress and coping strategy, and psychological distress and QOL. The possibility that there are relationships between stress coping strategies and some QOL status depending on some point of treatment.

Conclusions: The psychological distress during the treatment course of esophageal cancer is significantly associated with the coping strategies and QOL influenced by esophagectomy. This study can provide baseline information for identifying patients in need of psychological management and paves the way for larger clinical studies in the future.

Keywords: Predictive factors, Psychological distress, Coping, Health-related quality of life, Esophageal cancer

\section{Introduction}

Esophagectomy is the mainstay of curative treatment for esophageal cancer. However, it is a highly invasive surgery with serious postoperative complications and is one

\footnotetext{
* Correspondence: yu.ohkura107@gmail.com

'Section Division of Liaison Psychiatry and Palliative Medicine, Graduate

School of Medical and Dental Sciences, Tokyo Medical and Dental University, Tokyo, Japan

${ }^{2}$ Department of Gastroenterological Surgery, Toranomon Hospital, 2-2-2 Toranomon, Minato-ku, Tokyo 105-8470, Japan

Full list of author information is available at the end of the article
}

of the most complex interventions in gastrointestinal surgery. Recent advances in surgical techniques and perioperative intensive care have reduced the mortality and complications associated with esophagectomy [1], but it continues to be a challenging procedure with a reported mortality rate of $2.9-3.0 \%$ and a postoperative complication rate of $42.8-50.0 \%$ [2-4]. To meet the increasing demands of healthcare, environmental safety, and food quality, various methods and techniques have been reported as reviewers' comments. Among these,

C C The Author(s). 2020 Open Access This article is licensed under a Creative Commons Attribution 4.0 International License, which permits use, sharing, adaptation, distribution and reproduction in any medium or format, as long as you give appropriate credit to the original author(s) and the source, provide a link to the Creative Commons licence, and indicate if changes were made. The images or other third party material in this article are included in the article's Creative Commons licence, unless indicated otherwise in a credit line to the material. If material is not included in the article's Creative Commons licence and your intended use is not permitted by statutory regulation or exceeds the permitted use, you will need to obtain permission directly from the copyright holder. To view a copy of this licence, visit http://creativecommons.org/licenses/by/4.0/. The Creative Commons Public Domain Dedication waiver (http://creativecommons.org/publicdomain/zero/1.0/) applies to the data made available in this article, unless otherwise stated in a credit line to the data. 
not only the diagnosis and treatment of cancer but also the mental health of the patient itself is considered to be very important factors. Previous cross-sectional studies addressing emotional outcomes after resection for esophageal cancer have suggested that in 1-year survivors, $64 \%$ felt depressed and $80 \%$ expressed fear of death and metastasis [5]. These feelings may lead to discontinuation of additional chemotherapy or chemoradiotherapy after surgery. Moreover, a recent study identified a link between psychological state of anxiety or depression and an increased risk of death from cancer $[6,7]$. We described the relationship between psychological distress and health-related quality of life (HRQOL) on each point of the treatment of esophageal cancer in the past report [8]. In this past study, we analyzed risk factors of psychological distress "on each point of the treatment". However, when we pay attention to the chronological change of the Hospital Anxiety and Depression Scale (HADS) scores of each patient, it revealed that each patient had various mental agitation on each point and presented changes of HADS scores. The perioperative psychological stresses are usually influenced by postoperative course, physical conditions, and surrounding environment. Among these study subjects, two groups without change of the HADS scores exist during all points of the treatment. In other words, there are two types of patients who always feel stress and who always don't feel stress during the perioperative period [9]. This fact may suggest that these two patients groups (persistent high group and persistent low group) compared with other groups may have different coping styles and that it would be of great help to select these types of patients before starting treatment for the selection of candidates of efficient intervention. Therefore, we extracted these two groups in the present study as "persistent high (H-) group" and "persistent low (L-) group" and further adding the Mental Adjustment to Cancer scale (MAC) to our previously reported scores. If the characteristics of these two patient groups could be predicted, it would be possible to develop appropriate supportive interventions and reduce the psychological stress associated with esophageal cancer. The aim of this study was to clarify the patient characteristics presenting with psychological distress after esophagectomy compared with those without significant psychological distress at all 5 time points especially from the standpoints of personal coping styles and HRQOL.

\section{Methods}

\section{Definition of psychological distress}

The definition of psychological distress is a negative emotional state characterized by physical and/or emotional discomfort, pain, or anguish. In other words, it is psychological discomfort that interferes with your activities of daily living. The definition of "psychological distress" and the definition of "anxiety and depression" are not the same concepts. However, psychological distress can result in negative views of the environment, others, and the self. Sadness, anxiety, distraction, and symptoms of mental illness are manifestations of psychological distress $[10,11]$.

Several screening scales have been developed for the early detection of patients' psychological distress. The HADS score was developed by Zigmond and Snaith [12] and is well accepted to screen for psychiatric problems in medically ill patients. Generally, cancer patients whose psychological distress is mainly characterized by anxiety and depression can benefit from using HADS to detect this distress. And also, Kugaya et al. [9] proved the reliability and validity of the Japanese version of HADS, and the scale appeared to be simple, sensitive, and specific for screening for psychological distress in Japanese cancer patients. Therefore, we use HADS for screening for psychological distress in the present study.

\section{Study design and endpoints}

Participants comprised 152 consecutive patients who attended the outpatient clinic at the Department of Gastrointestinal Surgery at Toranomon Hospital between April 2017 and April 2019 were assessed for trial eligibility. Among these 152 patients, 102 patients who met the eligibility criteria participated in this study. The inclusion criteria were as follows: esophageal cancer including Siewert type I/II tumors of the esophagogastric junction; scheduled for subtotal esophagectomy; age 20 to 85 years; performance status 0 to 2 ; ability to provide informed consent; with previous oncological therapies for cancer; with previous surgical treatment for other diseases. We included patients $20-85$ years old. The reason is because patients may have senile dementia. Generally, about $3 \%$ of people between the ages of $65-74$ have dementia, 19\% between 75 and 84 , and nearly half of those over 85 years of age [13]. Therefore, we selected the patients $20-85$ years old.

The following exclusion criteria were applied: informed consent has not been obtained; on treatment for mental disorder; unsuitability for participation in the study because of psychological or physical stress in the opinion of the medical staff; refusal to undergo surgery; and requested nonsurgical treatment. The flow diagram of this study is shown in Fig. 1. Questionnaires were administered at 5 time points as follows: at the time of outpatient consultation before definitive diagnosis (time 1); at the time of determination of clinical stage before treatment; both of surgery and neoadjuvant therapy (time 2); about 2 weeks after surgery before final staging (time 3); at the time of determination of final staging (time 4); and at 3 months after surgery (time 5). The 


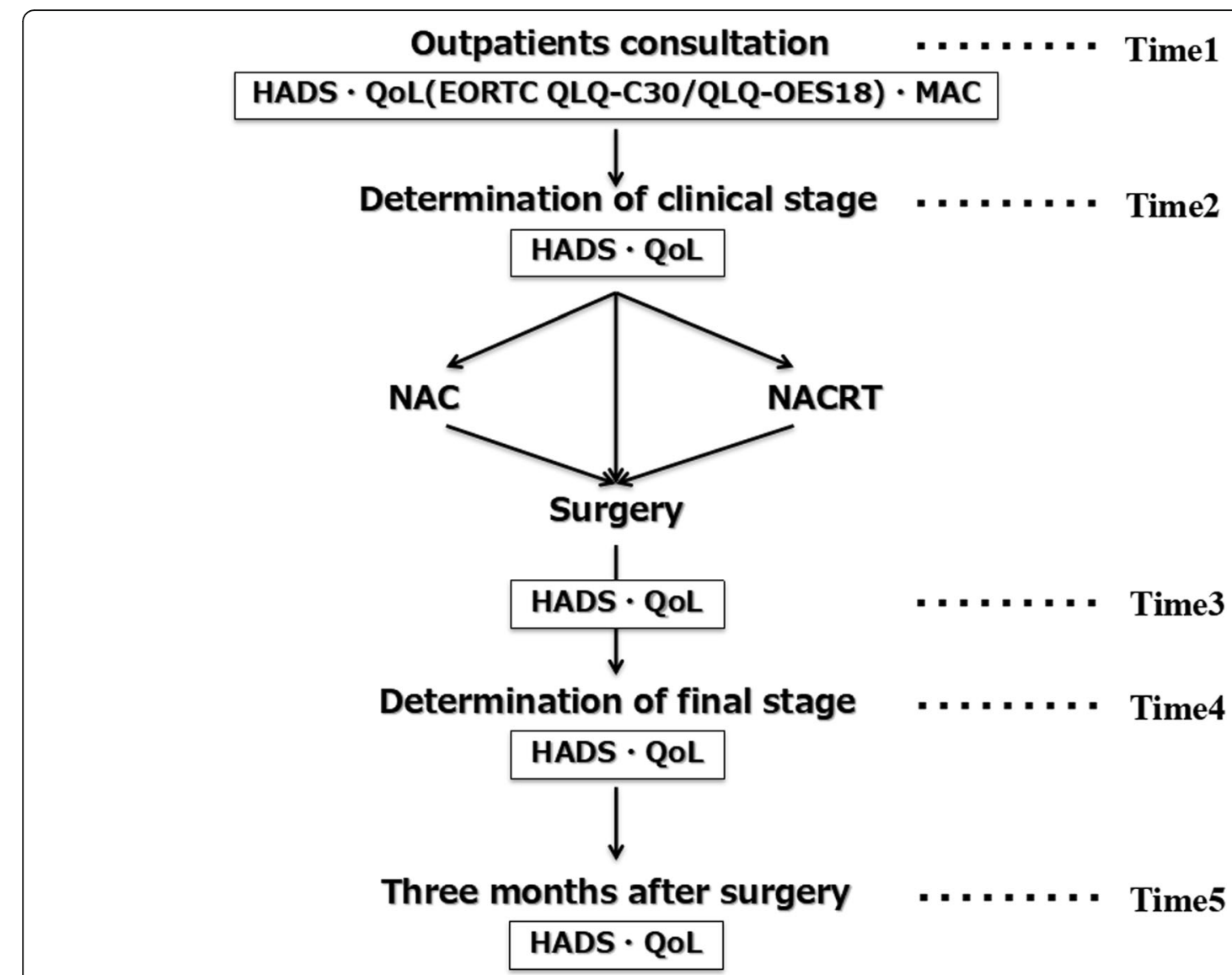

Fig. 1 Flow of patients through the study. Time 1, before definitive diagnosis; time 2, after determination of clinical stage; time 3, postoperatively before final staging; time 4, determination of final stage at 1 month after esophagectomy; time 5, 3 months after esophagectomy

questionnaires were administered in a fixed waiting room at our institution because we would like to perform the survey acquisition under the stable condition as much as possible. We selected three questionnaires; the Japanese version of HADS, the MAC scale, and the European Organization for Research and Treatment of Cancer Quality of Life Questionnaire Core 30/the oesophageal cancer-specific module (EORTC QLQ-C30/ OES18). The questionnaires (HADS/EORTC QLQ-C30/ OES18) were administered at all 5 time points, and the MAC scale was administered once, at time 1 . From the trends of HADS scores on each point of the treatment, we selected two groups: "persistent high HADS scores" and "persistent low HADS scores". Of 102 patients who met the eligibility criteria and participated in this study, 21 patients were allocated to the "persistent high $(\mathrm{H}-)$ group," and 38 patients were allocated to the "persistent low (L-) group" (Fig. 2). We investigated the characteristics of the H-group compared with the L-group especially from standpoints of personal coping styles and HRQOL. Esophageal cancer staging (clinical (c-)/pathological(p-) stage) is defined by the UICC TNM grading system, 7th edition; the sub-classifications based on the depth of invasion of the primary tumor (c-/p-T factors), lymph node involvement (c-/p-N factors), and extent of metastatic disease (c-/p-M factors) [14]. T factors classified the following; Tis: high-grade dysplasia, T1: invasion into the lamina propria, muscularis mucosae, or submucosa, T2: invasion into the muscularis propria, T3: invasion into the adventitia, T4a: invades resectable adjacent structures (pleura, pericardium, diaphragm), and T4b: invades unresectable adjacent structures (aorta, vertebral body, trachea). Similarly, $\mathrm{N}$ factors classified the following: N0: no regional lymph node metastases, N1: 1 to 2 positive regional lymph nodes, N2: 3 to 6 positive regional lymph nodes, and N3: 7 or more positive regional lymph nodes. $\mathrm{M}$ factors classified the following: M0: no distant metastases and M1: distant metastases [14]. All postoperative complications were graded using the Clavien-Dindo classification [15]; events more severe than grade $\geq$ III were recorded as complications. We chose this cut-off value on the basis of the Japanese version of HADS, which has been validated for Japanese patients with cancer [12]; a cut-off HADS total score $\geq 11$ has been recommended for identifying patients with potential adjustment disorder and 


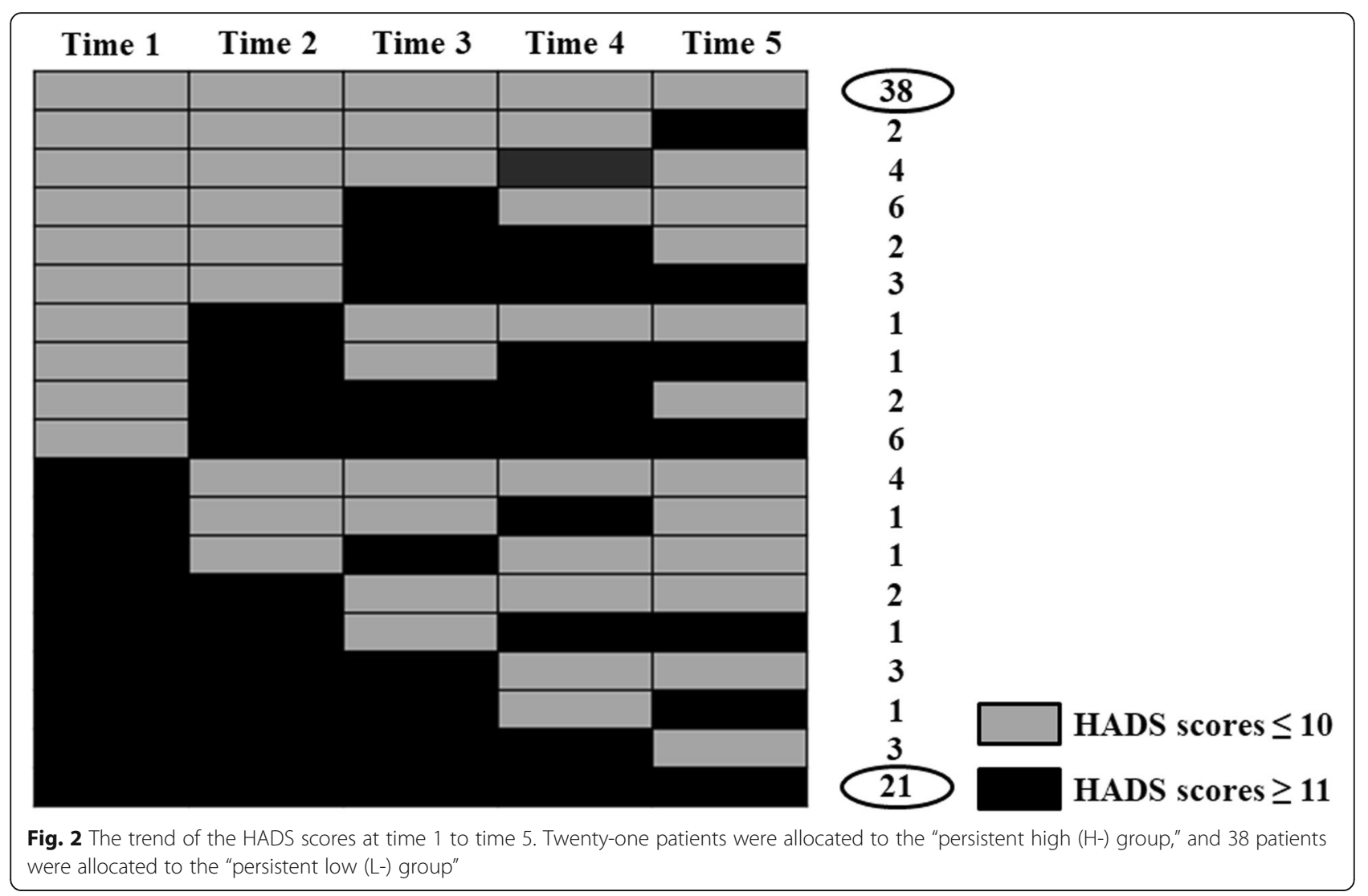

major depression [12]. We defined a total score $\geq 11$ as indicating psychological distress. The study protocol was approved by the institutional review boards of the Graduate School of Medical and Dental Sciences (approval number M2016-241) and Toranomon Hospital (approval number 1312) and registered with the UMIN Clinical Trials Registry (UMIN-CTR, R000033229). All procedures were conducted in accordance with the ethical standards of the Helsinki Declaration of 1975. Informed consent was obtained from all study participants at the time of the first outpatient appointment.

\section{Measures}

\section{Mental adjustment to cancer scale}

This scale (MAC scale) assesses the extent to which patients respond and adjust to their diagnosis of cancer and its treatment [16, 17]. It was developed as a selfrating questionnaire that would be patient-friendly and could be administered easily at busy oncology clinics. The scale includes five subscales that measure five types of response: fighting spirit ("I firmly believe that I will get better," 16 items); helpless/hopelessness ('I feel that life is hopeless," 6 items); anxious preoccupation ("I suffer great anxiety about it," 9 items); fatalism ("I've left it all to my doctors", 8 items); and avoidance ("I don't really believe I have cancer," 1 item). Each item is scored on a 4-point Likert scale (1, "definitely does not apply to me"; 4, "definitely applies to me"). Scores for the subscales are calculated by summing the answers for the assigned items. Generally, the coping skills do not greatly change in the short time; therefore, we did not measure the MAC scale at each time point $[18,19]$. This questionnaire was administered once, at time 1 , in this study.

\section{Hospital Anxiety and Depression Scale}

This scale (HADS) is a 14-item self-report questionnaire comprising of two subscales measuring symptoms of depression (HADS-D) and symptoms of anxiety (HADS-A) over the last week. Each subscale contains 7 items with scores ranging from 0 to 21 [11]. A total score of $\geq 11$ on either subscale indicates a definitive case. The Japanese version of HADS was back-translated by Kitamura [20] and its reliability and validity were confirmed by Kugaya et al. [9]. The questionnaire was administered at all 5 time points in this study.

\section{The European Organization for Research and Treatment of Cancer Quality of Life Questionnaire Core 30}

This questionnaire (EORTC QLQ-C30) is one of the most frequently used questionnaires to measure healthrelated quality of life in patients with cancer [21-23]. Its 
30 question items have been validated in several studies in various types of cancer. This score is a 30 -item questionnaire divided into five functional scales (physical, role, cognitive, emotional, and social), three symptom scales (fatigue, pain, and nausea/vomiting), six single-item questions (financial impact and various physical symptoms such as dyspnea, insomnia, anorexia, constipation, and diarrhea), and a global health status/quality of life. The items on the functional subscales are rated on a 4-point Likert scale (1, "not at all"; 4, "very much"). The raw subscale scores are transformed into a scale of $0-100(0$, poor QOL; 100, excellent QOL). The questionnaire was administered at all 5 time points in this study.

\section{EORTC QLQ-OES18 questionnaire}

The EORTC QLQ-OES18 is a self-reported 18-item questionnaire designed to assess health-related quality of life in patients undergoing a single treatment or a combination of treatments for esophageal cancer (i.e., esophagectomy, chemotherapy, radiotherapy, and/or chemoradiotherapy). It includes 12 items grouped into 4 symptom scales (dysphagia; 3 items, eating; 4 items, reflux; 2 items, and pain; 3 items) and 6 single items (trouble with swallowing saliva, choking, dry mouth, taste, cough, and speech). The time frame is "during the past week." All items are scored using a 4-point Likert scale (1, "not at all"; 4, "very much") and responses to the questionnaires were transformed into a 0-100 scale using EORTC guidelines [24]. The questionnaire was administered at all 5 time points in this study.

\section{Operative procedure for esophagectomy}

We perform esophagectomy with two-field or threefield lymphadenectomy depending on the degree of disease progression and the surgical risk involved. The operative thoracic approach entails video-assisted thoracoscopic surgery or thoracotomy and the abdominal approach involves hand-assisted laparoscopic surgery or open laparotomy depending on the individual case. We generally preserve the thoracic duct in patients with clinical stage I disease but resect it in those with clinical stage $\geq$ II disease for the purpose of lymphadenectomy [13]. However, we resected the thoracic duct even if the patient has clinical stage I disease when we suspect lymph node metastasis or have confirmed a metastasis in the lymph nodes along the bilateral recurrent laryngeal nerves on intraoperative pathologic analysis. However, we try to preserve the thoracic duct in patients at high risk of impaired hepatic or pulmonary function. A manually sutured esophagogastric or esophagoileal anastomosis in the neck is fashioned for all patients [25-29].

\section{Statistical analysis}

Differences between the two groups (H-group/ L-group) were tested for statistical significance using Fisher's exact test, the unpaired Student's $t$ test, the MannWhitney $U$ test, and Pearson's chi-squared test as appropriate.

Risk factors for psychological distress (H-group) were assessed by bivariate logistic regression analysis (Backward stepwise selection). The variables with a $p$ value less than 0.05 in univariate analysis were entered into bivariate logistic analysis. Odds ratios (ORs) and their $95 \%$ confidence intervals $(\mathrm{CIs})$ were calculated. A $p$ value less than 0.05 was considered statistically significant in bivariate logistic analysis. The correlations between the MAC scale and HRQOL status were assessed using Spearman's correlation coefficient and statistical significance was tested using Spearman's rank-sum test. The internal consistency (reliability) of each scale was estimated by Cronbach's alpha coeffcient [30]. A value of 0.70 or greater was considered acceptable for group comparison. All analysis was performed using SPSS for Windows software (version 19.0 J; IBM Corp., Armonk, NY).

\section{Results}

\section{Patient characteristics}

Fifty of the 152 patents considered for participation in the study were excluded because they had incomplete data $(n=27)$, declined to participate $(n=11)$, did not undergo esophagectomy $(n=11)$, or were receiving treatment for a psychiatric disorder $(n=1)$, leaving 102 patients for inclusion in the study. From the trends of HADS scores on each point of the treatment of esophageal cancer (Fig. 2), we selected two groups: "persistent high HADS scores" and "persistent low HADS scores". The characteristics of these patients in the two groups are shown in Table 1.

The mean age of L-group/H-group was 65.3/67.0 years, $86.8 \% / 85.7 \%$ were male, and median body mass index was $22.7 / 20.1$, respectively. Univariate analysis between the two groups showed a significant difference in the abdominal approach $(p=0.022)$, resection or preservation of the thoracic duct $(p=0.012)$, clinical $\mathrm{N}$ factors $(p=0.029)$, and MAC scales (fighting spirits; $p=0.001$, helpless/hopeless; $p<0.001$, anxious preoccupation; $\mathrm{p}<$ 0.001 , and fatalism; $p=0.008$ ).

\section{EORTC QLQ C-30 and psychological distress}

The trend of the scores of EORTC QLQ C-30 on each 5 points of the treatment of esophageal cancer is recorded in Fig. 3 and Table 2; data were compared between the two groups (H-group/L-group). Figure 3 a shows five functional subscales such as physical functioning, role functioning, emotional functioning, cognitive functioning, social functioning, and global health status/quality 
Table 1 Patient demographics and clinical characteristics

\begin{tabular}{llll}
\hline Variables & Persistent low group $(\mathbf{N}=\mathbf{3 8})$ & Persistent high group $(\mathbf{N}=\mathbf{2 1})$ & O \\
\hline Age, years, mean \pm SD & $64.5 \pm 8.079$ & $65.5 \pm 6.875$ & 69.2 \\
Male sex, \% & 86.8 & 85.7 & 81.4 \\
BMI, $\mathbf{k g} / \mathbf{m}^{2}$, median & $22.7(17.4-27.7)$ & $20.1(14.1-41.9)$ & 22.0 \\
$\begin{array}{l}\text { History of cancer } \\
\quad \text { Yes }\end{array}$ & 6 & 6 & 12 \\
$\quad$ No & 32 & 15 & 31 \\
History of surgery & & & 17 \\
$\quad$ Yes & 10 & 9 & 26 \\
$\quad$ No & 28 & 12 & 39 \\
History of alcohol consumption & & 18 & 4 \\
$\quad$ Yes & 34 & 3 &
\end{tabular}

\section{History of smoking}

$\begin{array}{ll}\text { Yes } & 3 \\ \text { No } & 7\end{array}$

\section{Brinkman index}

31

7

$632(0-2700)$

$<600$

$\geq 600$

\section{5}

23

Thoracic approach

VATS

Open

None: transhiatal

Abdominal approach

$\begin{array}{ll}\text { HALS } & 13 \\ \text { Open } & 6 \\ \text { Laparoscopic } & 19\end{array}$

Lymphadenectomy

DO

D2/3

Curability

RO

$\mathrm{R} 1 / 2$

Reconstruction

Gastric tube 25

lleocolic

Other

Thoracic duct

Resected

Preserved

Reconstruction route

Retrosternal
Posterior mediastinal

Preoperative treatment

1

2

5

\begin{tabular}{ll} 
Others $(\boldsymbol{N}=\mathbf{4 3})$ & $\boldsymbol{p}$ value \\
\hline $69.2 \pm 9.398$ & 0.680 \\
81.4 & 0.904 \\
$22.0(15.0-32.1)$ & 0.024 \\
& 0.243
\end{tabular}

12

31

17

26

39

4

$17 \quad 39$

$4 \quad 4$

$600(0-2760)$

$741(0-3040)$

0.975

0.544

$10 \quad 19$

$11 \quad 24$

0.234

$35 \square$

$17 \quad 36$

325

$13-11$

6

$19 \quad 3$

0.247

2

$19(5 / 14)$

0.665

20

0.780

29

12

2

4

0.193

23

$42(13 / 29)$

39

4

30

13

39

$8 \quad 2$ 
Table 1 Patient demographics and clinical characteristics (Continued)

\begin{tabular}{|c|c|c|c|c|}
\hline Variables & Persistent low group $(N=38)$ & Persistent high group $(N=21)$ & Others $(N=43)$ & $p$ value \\
\hline Yes & 16 & 5 & 27 & \\
\hline No & 22 & 16 & 16 & \\
\hline Operation time (min) & $592(350-734)$ & $597(415-703)$ & $574(213-774)$ & 0.795 \\
\hline Blood loss (mL) & $130(25-1175)$ & $274(25-1378)$ & $260(38-1094)$ & 0.067 \\
\hline G3 postoperative complications & & & & 0.855 \\
\hline Yes & 8 & 4 & 11 & \\
\hline No & 30 & 17 & 32 & \\
\hline cT factor (7th) & & & & 0.303 \\
\hline $1 \mathrm{a} / 1 \mathrm{~b}$ & $2 / 16$ & $1 / 5$ & $2 / 10$ & \\
\hline 2 & 12 & 6 & 7 & \\
\hline 3 & 6 & 6 & 18 & \\
\hline $4 a / 4 b$ & $0 / 2$ & $2 / 1$ & $2 / 4$ & \\
\hline \multicolumn{5}{|l|}{ cN factor (7th) } \\
\hline 0 & 25 & 7 & 13 & 0.029 \\
\hline 1 & 10 & 11 & 15 & \\
\hline 2 & 3 & 1 & 15 & \\
\hline 3 & 0 & 2 & 0 & \\
\hline cStage (7th) & & & & 0.109 \\
\hline $\mathrm{I}(\mathrm{IA}, \mathrm{B})$ & $12 / 9$ & $5 / 0$ & $10 / 2$ & \\
\hline$\|(\|A\| B)$, & $3 / 7$ & $1 / 7$ & $1 / 5$ & \\
\hline$\|||(I I A,\|I B\| I C)$, & $3 / 1 / 1$ & $1 / 1 / 4$ & $9 / 7 / 5$ & \\
\hline IV & 2 & 2 & 4 & \\
\hline p T factor (7th) & & & & 0.126 \\
\hline 0 & 2 & 2 & 2 & \\
\hline $1 a / 1 b$ & $5 / 19$ & $1 / 6$ & $3 / 8$ & \\
\hline 2 & 8 & 3 & 9 & \\
\hline 3 & 2 & 6 & 19 & \\
\hline $4 a / 4 b$ & $1 / 1$ & $2 / 1$ & $2 / 0$ & \\
\hline p N factor (7th) & & & & 0.276 \\
\hline 0 & 26 & 10 & 13 & \\
\hline 1 & 8 & 8 & 12 & \\
\hline 2 & 3 & 1 & 11 & \\
\hline 3 & 1 & 2 & 7 & \\
\hline p Stage (7th) & & & & 0.062 \\
\hline 0 & 2 & 1 & 0 & \\
\hline $\mathrm{I}(\mathrm{IA}, \mathrm{IB})$ & $18 / 2$ & $6 / 0$ & $8 / 3$ & \\
\hline$\|(\|A\| B)$, & $2 / 8$ & $0 / 3$ & $1 / 5$ & \\
\hline$\|I\|(I I A,\|I B\| I C)$, & $4 / 0 / 1$ & $3 / 1 / 5$ & $12 / 7 / 5$ & \\
\hline IV & 1 & 2 & 2 & \\
\hline Location of tumor & & & & 0.510 \\
\hline Cervical Esophagus & 2 & 1 & 1 & \\
\hline Upper thoracic esophagus & 5 & 4 & 10 & \\
\hline Middle thoracic esophagus & 15 & 12 & 19 & \\
\hline Lower thoracic esophagus & 7 & 3 & 10 & \\
\hline
\end{tabular}


Table 1 Patient demographics and clinical characteristics (Continued)

\begin{tabular}{|c|c|c|c|c|}
\hline Variables & Persistent low group $(N=38)$ & Persistent high group $(N=21)$ & Others $(N=43)$ & $p$ value \\
\hline Abdominal esophagus & 1 & 0 & 0 & \\
\hline Esophagogastric junction & 8 & 1 & 3 & \\
\hline \multicolumn{5}{|l|}{ MAC scale } \\
\hline Fighting spirit & $51.0(34-59)$ & $44.0(27-57)$ & $48.0(29-60)$ & 0.001 \\
\hline Helpless/hopeless & $7.0(6-16)$ & $12.0(6-24)$ & $10.0(6-16)$ & $<0.001$ \\
\hline Anxious preoccupation & $21.0(13-29)$ & $25.0(17-31)$ & $23.0(14-32)$ & $<0.001$ \\
\hline Fatalism & $18.0(8-29)$ & $21(12-30)$ & $22.0(13-28)$ & 0.008 \\
\hline Avoidance & $1.0(1-4)$ & $1.0(1-4)$ & $2.0(1-4)$ & 0.793 \\
\hline
\end{tabular}

Abbreviations: SD, standard deviation; BMI, body mass index; EORTC, European Organization for Research and Treatment; HADS, Hospital Anxiety and Depression Scale; VATS, video-assisted thoracoscopic surgery; HALS, hand-assisted laparoscopic surgery; MAC, mental adjustment to cancer; VATS, video-assisted thoracoscopic surgery

of life. In almost all the functional subscales on each 5 points, the H-group had a significantly lower and unhealthier level of functioning than the L-group. Figure 3 b shows 9 symptom scales such as fatigue, pain, nausea/ vomiting, pain, dyspnea, insomnia, anorexia, constipation, diarrhea, and financial impact. In almost all the symptom scales on each 5 points, the $\mathrm{H}$-group had a significantly higher level of symptomatology or problems than the L-group.

\section{EORTC QLQ OES-18 and psychological distress}

The trend of the scores of EORTC QLQ OES-18 on each 5 points is recorded in Fig. 4 and Table 2; data were compared between the two groups (H-group/Lgroup). Figure $4 \mathrm{a}$ and $\mathrm{b}$ show four symptom scales such as dysphagia, eating restriction, reflux, esophageal pain, and 6 single items such as trouble with swallowing saliva, choking, dry mouth, taste, cough, and speech. In almost all these scales on each 5 points, the $\mathrm{H}$-group had a significantly higher level of symptomatology or problems than the L-group.

\section{The relations between the stress coping strategies and QOL status}

There were correlations between the MAC scale and QOL status in some scales. The relationship between two variables is generally considered moderate when their correlation coefficient $(r)$ value is larger than 0.5 and also considered strong correlation when their $r$ value is larger than 0.7 [31]. The internal consistency (reliability) of each scale was estimated by Cronbach's alpha coefficient. A value of 0.70 or greater was considered acceptable for group comparison [30].

In this study, the $r$ values and Cronbach's alpha coefficient between the MAC scale (helpless/hopeless) and some variables of EORTC QLQ C-30/OES18 in several points of perioperative treatment were global (time $3 ; r$ $=-0.521(\alpha=0.501) ; p<0.001 /$ time $5 ; r=-0.576(\alpha=$
$0.558) ; p<0.001$ ), physical (time $5 ; r=-0.628(\alpha=$ $0.614) ; p<0.001$ ), emotional (time $1 ; r=-0.520(\alpha=$ $0.537) ; p<0.001 /$ time $3 ; r=-0.559(\alpha=0.560) ; p<$ $0.001)$, cognitive (time 3; $r=-0.558(\alpha=0.533) ; p<$ $0.001 /$ time $4 ; r=-0.524(\alpha=0.522) ; p<0.001)$, social (time $5 ; r=-0.542(\alpha=0.692) ; p<0.001)$, fatigue (time $2 ; r=0.530(\alpha=0.533) ; p<0.001 /$ time $5 ; r=0.583(\alpha=$ $0.562) ; p<0.001$ ), financial difficulty (time $4 ; r=0.509$ $(\alpha=0.516) ; p<0.001)$, OES18: eating restriction (time $3 ; r=-0.518(\alpha=0.516) ; p<0.001 /$ time $4 ; r=-0.501$ $(\alpha=0.733) ; p=0.001 /$ time $5 ; r=-0.573(\alpha=0.589)$; $p<0.001)$, and speech (time $5 ; r=0.503(\alpha=0.770) ; p$ $=0.001)$. Among these variables, there were moderate correlations between helpless/hopeless and eating restriction scale at time $4 /$ speech scale at time 5 considering Cronbach's alpha coefficient. On the other hand, there was a strong correlation between helpless/hopeless and role functioning scale at time $5(r=-0.752(\alpha=$ 0.752); $p<0.001)$.

\section{Discussion}

In this study, we evaluated psychological distress, stress coping strategy, and quality of life in patients with esophageal cancer from the time of the outpatient consultation (time 1) through to 3 months after esophagectomy (time 5). Our results showed that there are strong relationships between psychological distress and stress coping strategy, and psychological distress and quality of life. And also, this study suggests that there are relationships between stress coping strategies and some HRQOL status depending on the point of treatment.

We discussed and described that the clinical factors associated with psychological distress, focusing on the influence of HRQOL and the coping strategies of all patients with esophageal cancer on each point of the treatment [8]. In our past study, we analyzed risk factors of psychological distress "on each point of the treatment." However, when we pay attention to the chronological 


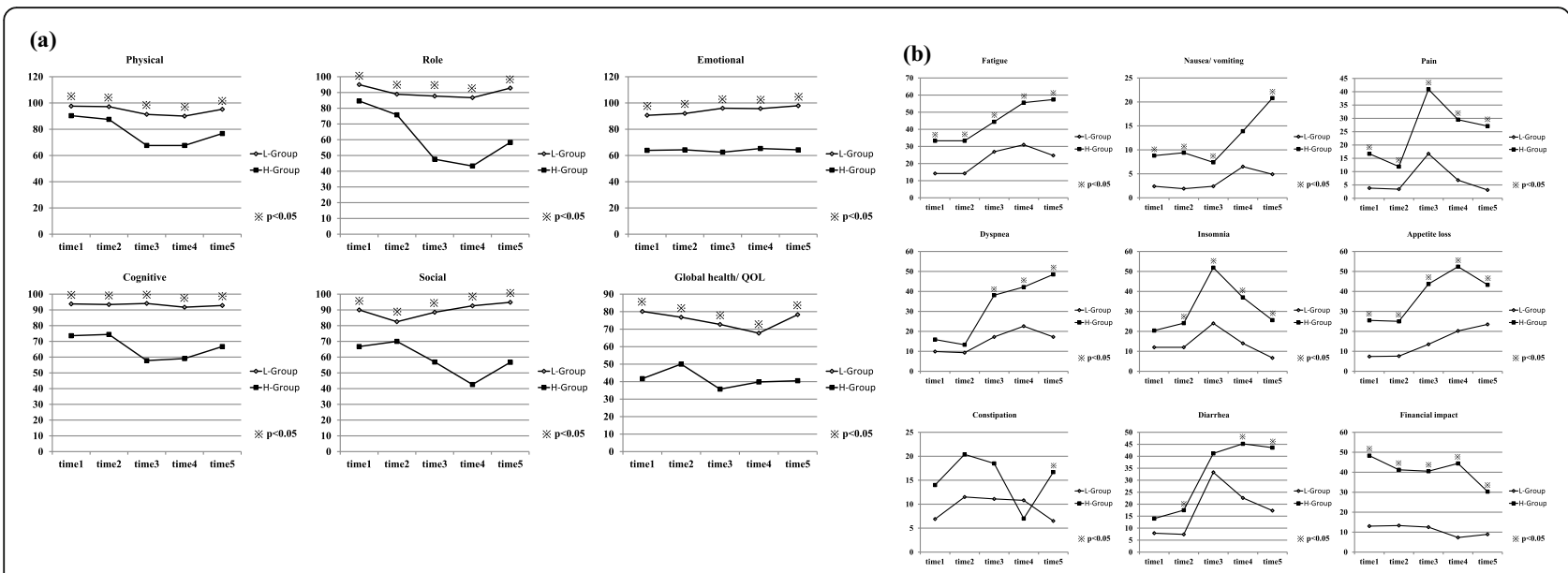

Fig. 3 The trend of the score of EORTC QLQ C-30. a Functioning scales: physical functioning, role functioning, emotional functioning, cognitive functioning, social functioning, and global health status/quality of life. b Symptom scales: fatique, pain, nausea/vomiting, pain, dyspnea, insomnia, anorexia, constipation, diarrhea, and financial impact

change of HADS scores of each patient, it revealed that each patient had various mental agitation on each point and presented changes of HADS scores, for example, 21 patients had "persistent high HADS scores", 38 patients had "persistent low HADS", 12 patinets had "High-Low", 11 patients had "Low-High", 15 patients had "Low-HighLow", and so on [8]. In the previous cross-sectional studies, the emotional outcomes after resection for esophageal cancer have suggested that in 1-year survivors, $64 \%$ felt depressed, and $80 \%$ expressed fear of death and metastasis [5]. Our present research revealed that about $60-70 \%$ of patients with esophageal cancer on each point of the treatment had psychological distress like the past report. However, only about $20 \%$ of patients had psychological distress at all times (persistent high HADS scores) during the treatment. Among these study subjects, two groups without change of the HADS scores exist during each point of the treatment. The possibility that these two patients group (persistent high group and persistent low group) compared with other groups may have a different coping style was guessed. Therefore, we extracted these two groups in the present study as "persistent high (H-) group" and "persistent low (L-) group" and further adding the coping skills questionnaire (MAC scale) to our previously reported scores.

\section{Patients background}

In the $\mathrm{H}$-group, there was a tendency for many cases to be resected with thoracic duct and to be positive for lymph node metastases. We generally resect the thoracic duct in patients with clinical stage $\geq$ II advanced disease for the purpose of lymphadenectomy. One possible explanation for these results is that the degree of disease progression may affect perioperative psychological distress.

\section{Coping skills}

In the past reports, the possibility that the coping skill improves the psychological distress had been discussed because there were significant associations between the coping skills and psychological distress [16, 17, 32]. However, it is still unclear whether the coping skills act on the psychological distress effectively in the various situations of the episode of the esophageal cancer treatment. In the present study, patients with a helpless/ hopeless response, anxious preoccupation, and fatalism were at increased risk of psychological distress, whereas those with a fighting spirit response were better able to adjust to their situations. And also, the results showed that the most beneficial response is fighting spirit and the most deleterious response may be helpless/hopeless. If we can grasp the stress coping-style of the patients with esophageal cancer even one investigation before treatment, it would be possible to develop appropriate supportive interventions and reduce the psychological stress, anxiety, and depressive feelings associated with esophageal cancer and surgery.

\section{HRQOL: EORTC QLQ C-30/OES18}

The emotional aspects such as worry and irritability have been shown to be associated with HRQOL [33]. And also, in the past reports, there was a significant association between the HRQOL and psychological distress [34-36]. We similarly described the psychological distress during the treatment course of esophageal cancer is significantly associated with HRQOL influenced by esophagectomy [8]. In the present study, although we selected only two groups of patients with or without psychological distress at all times for the perioperative period, we were able to describe that patients with psychological distress at all times had a significantly lower 
Table 2 The details of the QOL status: EORTC QLQ C-30 and EORTC OES-18

\begin{tabular}{|c|c|c|c|c|c|c|c|c|c|c|c|c|c|c|c|}
\hline & \multicolumn{3}{|c|}{ Time 1} & \multicolumn{3}{|c|}{ Time 2} & \multicolumn{3}{|c|}{ Time 3} & \multicolumn{3}{|c|}{ Time 4} & \multicolumn{3}{|c|}{ Time 5} \\
\hline & $\mathrm{H}$ & L & $\mathbf{P}$ & $\mathrm{H}$ & L & $\mathbf{P}$ & $\mathrm{H}$ & L & $\mathbf{P}$ & $\mathrm{H}$ & $\mathrm{L}$ & $P$ & $\mathrm{H}$ & L & $\mathbf{P}$ \\
\hline \multicolumn{16}{|l|}{ QLQ C-30 } \\
\hline Physical & 90.3 & 97.6 & 0.001 & 87.5 & 97.2 & 0.001 & 67.6 & 91.3 & $<0.001$ & 67.6 & 90.0 & $<0.001$ & 76.7 & 95.2 & $<0.001$ \\
\hline Role & 84.6 & 95.0 & 0.017 & 75.8 & 88.9 & 0.011 & 47.6 & 87.7 & $<0.001$ & 43.3 & 86.7 & $<0.001$ & 58.3 & 92.8 & $<0.001$ \\
\hline Emotional & 63.9 & 90.6 & $<0.001$ & 64.3 & 92.0 & $<0.001$ & 62.5 & 96.0 & $<0.001$ & 65.3 & 95.7 & $<0.001$ & 64.3 & 97.9 & $<0.001$ \\
\hline Cognitive & 73.6 & 93.8 & $<0.001$ & 74.4 & 93.4 & $<0.001$ & 57.8 & 94.1 & $<0.001$ & 59.1 & 91.7 & $<0.001$ & 66.7 & 92.8 & $<0.001$ \\
\hline Social & 66.7 & 90.0 & 0.001 & 70.0 & 82.5 & 0.045 & 56.9 & 88.5 & $<0.001$ & 42.6 & 92.6 & $<0.001$ & 56.7 & 94.8 & $<0.001$ \\
\hline Global health & 41.7 & 80.1 & $<0.001$ & 50.0 & 76.8 & 0.002 & 35.7 & 72.7 & $<0.001$ & 39.8 & 67.7 & $<0.001$ & 40.5 & 78.3 & $<0.001$ \\
\hline Fatigue & 33.3 & 14.2 & 0.001 & 33.3 & 14.2 & $<0.001$ & 44.4 & 26.9 & $<0.001$ & 55.6 & 31.0 & $<0.001$ & 57.4 & 24.7 & $<0.001$ \\
\hline Nausea & 8.8 & 2.4 & 0.014 & 9.4 & 1.9 & 0.006 & 7.4 & 2.4 & 0.040 & 13.9 & 6.5 & 0.132 & 20.8 & 4.9 & 0.005 \\
\hline Pain & 16.7 & 3.8 & 0.003 & 11.9 & 3.4 & 0.017 & 40.9 & 16.7 & $<0.001$ & 29.5 & 6.8 & $<0.001$ & 27.1 & 3.1 & $<0.001$ \\
\hline Dyspnea & 15.9 & 9.9 & 0.187 & 13.3 & 9.3 & 0.383 & 38.1 & 17.2 & 0.003 & 42.2 & 22.6 & 0.017 & 48.5 & 17.2 & $<0.001$ \\
\hline Insomnia & 20.4 & 12.0 & 0.182 & 24.1 & 12.0 & 0.034 & 51.9 & 24.0 & 0.001 & 37.0 & 14.0 & $<0.001$ & 25.6 & 6.7 & 0.002 \\
\hline Appetite loss & 25.5 & 7.4 & 0.002 & 25.0 & 7.6 & 0.003 & 43.7 & 13.5 & 0.001 & 52.4 & 20.2 & 0.001 & 43.3 & 23.5 & 0.032 \\
\hline Constipation & 14.0 & 6.9 & 0.149 & 20.4 & 11.5 & 0.140 & 18.5 & 11.1 & 0.212 & 7.0 & 10.8 & 0.418 & 16.7 & 6.5 & 0.047 \\
\hline Diarrhea & 14.0 & 7.9 & 0.182 & 17.5 & 7.4 & 0.031 & 41.2 & 33.3 & 0.389 & 45.2 & 22.6 & 0.015 & 43.6 & 17.3 & 0.002 \\
\hline Financial & 48.3 & 13.0 & $<0.001$ & 41.2 & 13.3 & $<0.001$ & 40.5 & 12.5 & 0.001 & 44.4 & 7.3 & $<0.001$ & 30.3 & 8.9 & 0.003 \\
\hline \multicolumn{16}{|l|}{ OES 18} \\
\hline Dysphagia & 63.5 & 94.8 & 0.003 & 82.2 & 96.1 & 0.002 & 62.5 & 92.6 & $<0.001$ & 64.4 & 84.8 & $<0.001$ & 63.5 & 89.9 & 0.328 \\
\hline Eating & 58.3 & 93.5 & $<0.001$ & 68.3 & 94.3 & 0.001 & 59.3 & 83.9 & $<0.001$ & 41.7 & 74.3 & $<0.001$ & 47.2 & 78.8 & 0.010 \\
\hline Reflux & 87.5 & 96.2 & 0.004 & 87.8 & 96.2 & 0.010 & 86.1 & 94.6 & 0.040 & 81.7 & 90.0 & 0.122 & 75.9 & 91.0 & 0.161 \\
\hline Eso. pain & 82.7 & 98.0 & $<0.001$ & 87.5 & 97.4 & 0.005 & 71.4 & 93.8 & 0.001 & 75.9 & 94.0 & 0.005 & 74.1 & 97.5 & 0.010 \\
\hline Saliva & 90.0 & 97.4 & 0.033 & 90.5 & 100 & 0.026 & 80.4 & 95.4 & 0.002 & 74.5 & 91.7 & 0.002 & 91.1 & 96.8 & 0.442 \\
\hline Choking & 86.7 & 96.5 & 0.011 & 88.3 & 97.4 & 0.012 & 74.5 & 90.7 & 0.003 & 70.6 & 88.9 & 0.001 & 73.8 & 89.2 & 0.105 \\
\hline Dry mouth & 70.8 & 88.0 & 0.009 & 70.8 & 85.2 & 0.031 & 58.8 & 79.3 & 0.007 & 57.1 & 86.0 & 0.002 & 69.7 & 92.5 & 0.442 \\
\hline Taste & 87.0 & 95.4 & 0.073 & 76.2 & 91.7 & 0.050 & 66.7 & 88.6 & $<0.001$ & 61.5 & 89.6 & 0.001 & 69.4 & 89.7 & 0.161 \\
\hline Cough & 90.0 & 94.6 & 0.249 & 86.0 & 94.6 & 0.053 & 64.1 & 78.1 & 0.092 & 53.3 & 79.0 & 0.009 & 60.6 & 85.1 & 0.721 \\
\hline Speech & 91.7 & 94.4 & 0.469 & 95.0 & 94.4 & 0.899 & 62.2 & 81.2 & 0.010 & 47.6 & 72.6 & 0.004 & 56.7 & 92.2 & 0.721 \\
\hline
\end{tabular}

and unhealthier level of functioning in almost all the functional scales, and a higher level of symptomatology or problems in almost all the symptom scales than the patients without psychological distress. The symptoms that patients with esophageal cancer have would be affected physically by esophageal cancer itself such as "Fatigue," "Nausea/vomiting," "Pain," and "Appetite loss" in EORTC QLQ C-30 and "Dysphagea," "Eating restriction, " "Reflux," and "Esophageal pain" in OES 18 especially at time 1/time 2 (preoperation). And also, the symptoms that patients after esophagectomy have would be affected physically by surgery itself such as "Fatigue," "Nausea/vomiting," "Pain," "Dyspnea," "Insomnia," and "Appetite loss" in EORTC QLQ C-30 and "Dysphagea," "Eating restriction," "Reflux," "Esophageal pain," "Choking," "Dry mouth," "Taste," "Cough," and "Speech" in OES 18 especially at time 3/time 4 (postoperation). Through all episodes of treatment, "The financial difficulties status," "Role functioning," and "Social status" was a significant factor for psychological distress. We could guess that the patients have large anxiety about treatment strategies and costs of esophageal cancer treatments particularly in the era of multimodal treatment which requires more time and cost. Also, patients feel so sorry to trouble his or her family because of their physical problems such as "eating difficulties." Except for our past [8] and present reports, there is no report which described more detail about the association between HADS and HRQOL.

The relations between coping skills and HRQOL

It is usually expected that patients with advanced diseases have a tendency of higher HADS scores and more impaired QOL. It is also suggested that those who were informed of very advanced or non-curable disease status revealed surgically or pathologically would have higher 

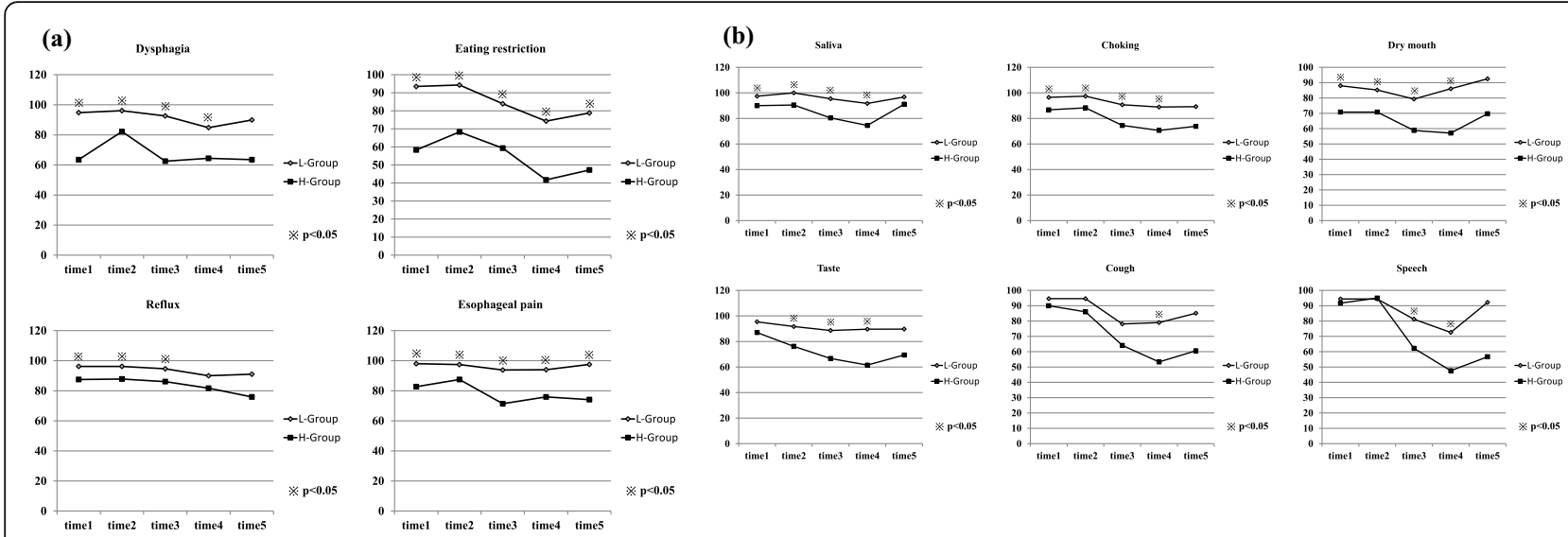

Fig. 4 The trend of the score of EORTC QLQ OES18. a Symptom scales: dysphagia, eating restriction, reflux, esophageal pain. b Single items: trouble with swallowing saliva, choking, dry mouth, taste, cough, and speech

HADS scores and more impaired QOL after the information. Psychological distress, QOL, disease status, and its recognition by the patient, all these factors should correlate with each other and their causal relationships are complicated and can vary with time course. Patients included neither in $\mathrm{H}$-group nor in L-group, who showed chronological changes of HADS score levels, might have been influenced by the information provided through the treatment course. Further examination will be necessary about these strategies in the future. However, this study showed that more than $50 \%$ of the patients consistently had high or low HADS scores throughout the course of the treatment of their esophageal cancers, and surprisingly, there was little difference in clinical characteristics between the two groups, but the two groups had a clear difference in 4 out of 5 subscales of the MAC scale measured preoperatively. Therefore, at least for such half or more patients, preoperative MAC scale evaluation can be useful in selecting patients who can benefit by appropriate supportive interventions on their coping skills and it may reduce their psychological distress and hopefully improve their QOL.

There is no report which described the association between the MAC scale and HRQOL in the past. Therefore, it is still unclear whether the Coping skills act on the HRQOL effectively in the various situations of the episode of the esophageal cancer treatment. We investigated in more detail that the association between stress coping strategy and HRQOL on each point of the treatment of esophageal cancer. As described above, most of the symptoms that patients with esophageal cancer have would be affected physically by esophageal cancer itself and the treatment including an operation. However, there is a possibility that coping skills would be related to the emergence of postoperative symptoms. In fact, there was a correlation between the MAC scale and
QOL status in some scales such as helpless/hopelesseating restriction, speech, and role functioning in the present study.

The major limitations for this study are the singlecenter design and the small sample size investigated. An external validation study involving a sufficient number of patients would be needed to confirm our observations; a multicenter study with a larger number of cases is also warranted. We selected four questionnaires according to the past reports, but using these questionnaires might not be sufficient to fulfill the purpose of our study. There might be other more suitable questionnaires to satisfy this study purpose. There were also several factors potentially associated with psychological distress related to treatment for esophageal cancer that could not be controlled for in this study. Nevertheless, our results confirm that the risk of psychological distress can be estimated reasonably accurately using the clinical factors investigated in this study. Prospective accumulation of clinical data using this study could provide important information for better psychological management of patients undergoing treatment for esophageal cancer.

\section{Conclusion}

This study showed that the psychological distress during the treatment course of esophageal cancer is significantly associated with the coping strategies and HRQOL influenced by esophagectomy. The current results may warrant prospective intervention through enhanced recovery after surgery to better manage patients undergoing highly invasive procedures for esophageal cancer. This study uses basic clinical data and may provide the baseline information for risk stratification for psychological management and for future clinical studies in these patients. 


\section{Abbreviations}

HADS: Hospital Anxiety and Depression Scale; MAC scale: Mental Adjustment to Cancer scale; EORTC QLQ-C30/OES18: the European Organization for Research and Treatment of Cancer Quality of Life Questionnaire Core 30/the oesophageal cancer-specific module; HRQOL: Health-related quality of life

\section{Authors' contributions}

Yu Ohkura, Kanako Ichikura, and Eisuke Matsushima designed the study, wrote the manuscript, revised it critically for important intellectual content, and approved its content; Yu Ohkura, Junichi Shindoh, Kanako Ichikura, Masaki Ueno, Harushi Udagawa and Eisuke Matsushima created the study materials and recruited patients. All authors read and approved the final manuscript.

\section{Availability of data and materials}

Some advocates of clinical data sharing are keen for data to be shared in agreed, standardized formats to facilitate its automated reuse for statistical analysis.

\section{Ethics approval and consent to participate}

The study protocol was approved by the institutional review boards of the Graduate School of Medical and Dental Sciences (approval number M2016 241) and Toranomon Hospital (approval number 1312) and registered with the UMIN Clinical Trials Registry (UMIN-CTR, R000033229). All procedures were conducted in accordance with the ethical standards of the Helsinki Declaration of 1975. Informed consent was obtained from all study participants at the time of the first outpatient appointment.

\section{Consent for publication}

Informed consent was obtained from these patients for publication of this report.

\section{Competing interests}

None declared.

\section{Author details}

1 Section Division of Liaison Psychiatry and Palliative Medicine, Graduate School of Medical and Dental Sciences, Tokyo Medical and Dental University, Tokyo, Japan. ²Department of Gastroenterological Surgery, Toranomon Hospital, 2-2-2 Toranomon, Minato-ku, Tokyo 105-8470, Japan. ${ }^{3}$ Okinaka Memorial Institute for Medical Research, Tokyo, Japan. ${ }^{4}$ Department of Health Science School of Allied Health Sciences, Kitasato University, Tokyo, Japan.

\section{Received: 15 August 2020 Accepted: 23 November 2020}

Published online: 09 December 2020

\section{References}

1. Takeuchi H, Saikawa Y, Oyama T, Ozawa S, Suda K, Wada N, et al. Factors influencing the long-term survival in patients with esophageal cancer who underwent esophagectomy after chemoradiotherapy. World J Surg. 2010;34: 277-84.

2. Takeuchi H, Miyata H, Gotoh M, Kitagawa $Y$, Baba H, Kimura W, et al. A risk model for esophagectomy using data of 5354 patients included in a Japanese nationwide web-based database. Ann surg. 2014;260:259-66.

3. Birat D, Brian SD, John GH, Brett CS, John TV, James PD. Patient and perioperative predictors of morbidity and mortality after esophagectomy: American College of Surgeons National Surgical Quality Improvement Program (ACS-NSQIP), 2005-2008. J Gastrointest Surg. 2010;14:1492-501.

4. Ohkura $Y$, Miyata $H$, Konno $H$, et al. Development of a model predicting the risk of eight major postoperative complications after esophagectomy based on 10826 cases in the Japan National Clinical Database. J Surg Oncol. 2020; 121:313-21.

5. Hellstadius $Y$, Lagergren $P$, Lagergren J, et al. Aspects of emotional functioning following oesophageal cancer surgery in a population-based cohort study. Psycho-Oncology. 2015;24:47-53.

6. Batty GD, Russ TC, Stamatakis E, et al. Psychological distress in relation to site specific cancer mortality: pooling of unpublished data from 16 prospective cohort studies. BMJ. 2017;356:j108.

7. Bouras G, Markar SR, Burns EM, et al. Linked hospital and primary care database analysis of the incidence and impact of psychiatric morbidity following gastrointestinal cancer surgery in England. Ann Surg. 2016;264:939.

8. Ohkura Y, Ichikura K, Shindoh J, et al. Relationship between psychological distress and health-related quality of life at each point of the treatment of esophageal cancer. Esophagus. 2020;17:312-22.

9. Kugaya A, Akechi T, Okuyama T, et al. Screening for psychological distress in Japanese cancer patients. Jpn J Clin Oncol. 1998;28(5):333-8.

10. Horwitz AV. Distinguishing distress from disorder as psychological outcomes of stressful social arrangments. Health. 2007;11:273-89.

11. Ridner SH. Psychological distress: concept analysis. Journal of Advanced Nursing. 2004;45(5):536-45.

12. Zigmond AS, Snaith RP. The hospital anxiety and depression scale. Acta Psychiatr Scand. 1983;67:361-70.

13. Umphred, Darcy. Neurological rehabilitation (6th ed.). 2012: 838

14. Sobin LH, Gospodarowicz MK, Wittekind C. International Union Against Cancer. Oesophagus including oesophagogastric junction. "TNM classification of malignant tumours". West Sussex,UK: Wiley-Blackwell; 2009. p. 66-72.

15. Dindo D, Demartines N, Clavien PA. Classification of surgical complications: a new proposal with evaluation in a cohort of 6336 patients and results of a survey. Ann Surg. 2004;240:205-13.

16. Osborne RH, Elsworth GR, Kissane DW, et al. The Mental Adjustment to Cancer (MAC) Scale: replication and refinement in 632 breast cancer patients. Psychological Medicine. 1999;29:1335-45.

17. Watson M, Greer S, Young J, et al. Development of a questionnaire measure of adjustment to cancer: the MAC scale. Psychological Medicine. 1988;18: 203-9.

18. Aldwin CM, Revenson TA. Does coping help? A reexamination of the relation between coping and mental health. Journal of Personality and Social Psychology. 1987;53:337-48.

19. Sandler IN, Tein JY, Mehta P, et al. Coping efficacy and psychological problems children of divorce. Child Development. 2000;71:1099-118.

20. Kitamura T. Hospital Anxiety and Depression Scale. Seisinka Sindangaku 1993; 4: 371-372(Japanese).

21. Tabano M, Condosta D, Coons M. Symptoms Affecting quality of life in women with gynaecologic cancer. Seminarsin Ontology Nursing. 2002;18(3): 223-30

22. Fayers PM, Aaronson NK, Bjordal K, et al. The EORTC QLQ C30 scoring manual (3rdEdition). Published by: European Organisation for Research and Treatment of Cancer, Brussels. 2001

23. Birhanu AA, Shiferaw N, Lukman $Y$, et al. Reliability and validity of Amharic version of EORTC QLQ-C 30 questionnaire among gynecological cancer patients in Ethiopia. PLoS ONE. 2016;11:e0157359.

24. Fujita T, Okada N, Sato T, et al. Translation, validation of the EORTC esophageal cancer quality-of-life questionnaire for Japanese with esophageal squamous cell carcinoma: analysis in thoraco-laparoscopic esophagectomy versus open esophagectomy. Japanese Journal of Clinical Oncology. 2016;46(7):615-21

25. Udagawa $H$, Akiyama $H$. Surgical treatment of esophageal cancer: Tokyo experience of the three-field technique. Dis Esophagus. 2001;14:110-4.

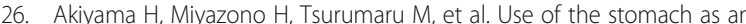
esophageal substitute. Ann Surg. 1978;188:606-10.

27. Udagawa $\mathrm{H}$, Ueno $\mathrm{M}$, Kinoshita $\mathrm{Y}$. Rationale for video-assisted radical esophagectomy. Gen Thorac Cardiovasc Surg. 2009;57:127-31.

28. Udagawa $H_{\text {, Ueno }} \mathrm{M}$, Shinohara $\mathrm{H}$, et al. The importance of grouping of lymph node stations and rationale of three-field lymphoadenectomy for thoracic esophageal cancer. J Surg Oncol. 2012 Nov;106(6):742-7.

29. Udagawa $H$, Ueno M, Shinohara $H$, et al. Should lymph nodes along the thoracic duct be dissected routinely in radical esophagectomy? Esophagus. 2014;11:204-10.

30. Cronbach LJ. CoeYcient alpha and the internal structure of tests. Psychometrika. 1951;16:297.

31. Nguyen NT, Gaol FL, Hong TP, et al. Intelligent Information and Database Systems: 11th Asian Conference, ACIIDS 2019, Yogyakarta, Indonesia, April 8-11, 2019, Proceedings, Part II. 2019: 110

32. Akechi T, Kugaya A, Okamura $H$, et al. Predictive factors for psychological distress in ambulatory lung cancer patients. Support Care Cancer. 1998;6: 281-6.

33. De Boer AG, Genovesi PI. Quality of life in long-term survivors after curative transhiatal oesophagectomy for oesophageal carcinoma. Br J Surg. 2000;87: $1716-21$. 
34. Subramaniam S, Kong YC, Chinna $\mathrm{K}$, et al. Health-related quality of life and psychological distress among cancer survivors in a middle-income country. Psycho-oncology. 2018:1-8.

35. Lagergren P, Avery KNL, Hughes R, et al. Health-related Quality of life among patients cured by surgery for esophageal cancer. Cancer. 2007;110 686-93.

36. Hulbert-Williams N, Neal R, Morrison V, et al. Anxiety, depression and quality of life after cancer diagnosis: what psychosocial variables best predict how patients adjust? Psycho-Oncology. 2012;21:857-67.

\section{Publisher's Note}

Springer Nature remains neutral with regard to jurisdictional claims in published maps and institutional affiliations.

Ready to submit your research? Choose BMC and benefit from:

- fast, convenient online submission

- thorough peer review by experienced researchers in your field

- rapid publication on acceptance

- support for research data, including large and complex data types

- gold Open Access which fosters wider collaboration and increased citations

- maximum visibility for your research: over $100 \mathrm{M}$ website views per year

At $B M C$, research is always in progress.

Learn more biomedcentral.com/submissions 